University of Nebraska - Lincoln

DigitalCommons@University of Nebraska - Lincoln

January 1970

\title{
Projected Hartree Product Wavefunctions. V. Density Matrices and Operators Containing Spin
}

Gordon A. Gallup

UNL,ggallup1@unl.edu

Follow this and additional works at: https://digitalcommons.unl.edu/physicsgallup

Part of the Physics Commons

Gallup, Gordon A., "Projected Hartree Product Wavefunctions. V. Density Matrices and Operators Containing Spin" (1970). Gordon Gallup Publications. 19.

https://digitalcommons.unl.edu/physicsgallup/19

This Article is brought to you for free and open access by the Research Papers in Physics and Astronomy at DigitalCommons@University of Nebraska - Lincoln. It has been accepted for inclusion in Gordon Gallup Publications by an authorized administrator of DigitalCommons@University of Nebraska - Lincoln. 


\title{
Projected Hartree Product Wavefunctions. V. Density Matrices and Operators Containing Spin*
}

\author{
G. A. Galluup \\ Department of Chemistry, University of Nebraska, Lincoln, Nebraska 68508
}

(Received 7 April 1969)

\begin{abstract}
Formulas are given to allow the calculation of the various spin components of the first-order density and transition density matrices from one spatial component of a pure spin-state wavefunction and certain reduced operators. Similar expressions are given for the second-order density for singlet and doublet systems. These results are useful for the calculation of matrix elements of operators containing the spin.
\end{abstract}

\section{INTRODUCTION}

In two recent papers, $\mathrm{I}^{1}$ and $\mathrm{II}^{2}$ of this series, the author has given a discussion of the use of the symmetric groups in determining eigenfunctions of the spin angular momentum operator for an application to the projected Hartree-Fock problem. These papers may be consulted for general references.

It is shown in I and II that matrix elements of spinfree operators may be evaluated without knowledge of the complete wavefunction, but knowing only the part associated with one primitive idempotent from the group algebra of the appropriate symmetric group. This idempotent $E^{(\mu)}(\gamma)$ depends on the Hamiltonian operator, $H$ (which in our approximation is considered spin free) through the numbers, $\gamma$, which must be adjusted to minimize

$$
W=\left\langle\phi\left|H E^{(\mu)}(\gamma)\right| \phi\right\rangle /\left\langle\phi\left|E^{(\mu)}(\gamma)\right| \phi\right\rangle .
$$

The $\gamma$ also depend, of course, on the choice for $\phi$. That only $E^{(\mu)}(\gamma) \phi$ is necessary for determination of the energy has also been emphasized in the work of Matsen et $a .^{3}$

The analysis which allows the energy to be calculated from Eq. (1) depends essentially on the spin-free nature of the Hamiltonian used and can be applied unchanged to the determination of the matrix elements of any spin-free operator. However, the method breaks down for the operator of a quantity depending on the spin. This type of operator is by no means unimportant, and we led to its consideration for an analysis of various magnetic phenomena, spin-orbit coupling, etc.

The problem of determining the matrix elements of operators can be transformed to the problem of determining certain reduced density and transition density matrices. ${ }^{4}$ The $p$ th-order reduced density matrix for an

\footnotetext{
* Based in part on work done while the author was on leave from the University of Nebraska at the Quantum Theory Project, University of Florida, Gainesville, Fla. The leave was made possible by the University of Nebraska Research Council and the National Science Foundation.

1 G. A. Gallup, J. Chem. Phys. 48, 1752 (1968)

${ }^{2}$ G. A. Gallup, J. Chem. Phys. 50, 1206 (1969).

${ }^{3}$ F. A. Matsen, Advan. Quantum Chem. 1, 59 (1964).

4 P.-O. Löwdin, Phys. Rev. 97,1474 (1955).
}

$n$-electron system is usually defined as

$$
\begin{aligned}
& \Gamma^{p}\left(x_{1}, x_{2}, \cdots, x_{p} \mid x_{1}{ }^{\prime}, x_{2}{ }^{\prime}, \cdots, x_{p}{ }^{\prime}\right) \\
&=\left(\begin{array}{c}
n \\
p
\end{array}\right) \int \Phi\left(x_{1}{ }^{\prime} x_{2}{ }^{\prime} \cdots x_{p}{ }^{\prime} x_{p+1} \cdots x_{n}\right)^{*} \\
& \times \Phi\left(x_{1} \cdots x_{p}, x_{p+1} \cdots x_{n}\right) d x_{p+1} \cdots d x_{n},
\end{aligned}
$$

where $\Phi$ is the complete antisymmetric wavefunction for the state in question. The transition density is similarly defined.

In order to apply Eq. (2) it is necessary to use the complete wavefunction. This is a purely group theoretic problem once $E^{(\mu)}(\gamma) \phi$ is known. If we consider $E^{(\mu)}(\gamma)$ (which is a primitive Hermitian idempotent of the appropriate symmetric group algebra) to be $e_{11}{ }^{(\mu)}$ of the matric basis corresponding to the irreducible representation determined by the partition $(\mu)$, the shifting operators $e_{i 1}^{(\mu)}, i=1,2, \cdots, f_{\mu}$, exist ${ }^{5}$ and may be determined, if no other method is available, by a sort of Schmidt orthogonalization procedure. Corresponding to $e_{i 1}^{(\mu)}$ there is a set $e_{i 1}^{(\bar{\mu})}$ for the conjugate partition $(\bar{\mu})$. If $\omega$ is a product of spin functions

$$
\omega=\alpha_{1} \alpha_{2} \cdots \alpha_{n-k} \beta_{n-k+1} \cdots \beta_{n}
$$

such that $e_{11}^{(\bar{\mu})} \omega$ does not vanish,

$$
\Phi=\sum_{i=1}^{f \mu}\left(e_{i 1}^{(\mu)} \phi\right)\left(e_{i 1}^{(\bar{\mu})} \omega\right)
$$

gives the complete (unnormalized) antisymmetric wavefunction for Eq. (2). However, the determination of the $e_{i 1}^{(\mu)}$ is far from a trivial problem in general, and we wish to circumvent it if possible. So we shall investigate the possibility of determining $\Gamma^{(p)}$ from $E^{(\mu)}(\gamma) \phi$ alone, and we shall restrict the discussion to the cases $p=1,2$. It is not a priori obvious, of course, that our program is possible, but we shall see that $E^{(\mu)}(\gamma) \phi$ is sufficient for the case $p=1$, and can give for the case $p=2$ a form of the density matrix which is sufficient for a certain restricted class of two particle

${ }^{5}$ D. E. Littlewood, Theory of Group Characters (Oxford University Press, London, 1940). 
operators involving the spin. We shall discuss the restriction later.

Matsen and Cantu ${ }^{6}$ have made progress in the solution of this problem by giving a method for determining what is essentially the spin density for spin-free wavefunctions. We shall take a different approach to the problem, one which appears more easily generalizable.

\section{DENSITY OPERATORS AND THEIR ALGEBRAIC PROPERTIES}

We shall discuss only the density matrices since the transition densities are obtained by an easy generalization.

$\mathrm{McWeeny}^{7}$ and McWeeny and Mizuno ${ }^{8}$ have given a careful discussion of density matrices and show that they have the forms

$$
\begin{aligned}
& \Gamma^{(1)}\left(x_{1} \mid x_{1}{ }^{\prime}\right)=\gamma\left(x_{1} \mid x_{1}{ }^{\prime}\right)=P^{\alpha \alpha}\left(p_{1} \mid \rho_{1}{ }^{\prime}\right) \alpha_{1} \alpha_{1}, * \\
& +P^{\beta \beta}\left(\rho_{1} \mid \rho_{1}^{\prime}\right) \beta_{1} \beta_{1}{ }^{*}, \\
& \Gamma^{(2)}\left(x_{1} x_{2} \mid x_{1}{ }^{\prime} x_{2}{ }^{\prime}\right)=\Gamma\left(x_{1} x_{2} \mid x_{1}{ }^{\prime} x_{2}^{\prime}\right) \\
& =P^{\alpha \alpha \alpha \alpha}\left(\rho_{1} \rho_{2} \mid \rho_{1}{ }^{\prime} \rho_{2}{ }^{\prime}\right) \alpha_{1} \alpha_{2} \alpha_{1},{ }^{*} \alpha_{2},{ }^{*} \\
& +P^{\alpha \beta \alpha \beta}\left(\rho_{1} \rho_{2} \mid \rho_{1}^{\prime} \rho_{2}^{\prime}\right) \alpha_{1} \beta_{2} \alpha_{1}{ }^{*} \beta_{2}, * \\
& +P^{\beta \alpha \beta \alpha}\left(\rho_{1} \rho_{2} \mid \rho_{1}^{\prime} \rho_{2}^{\prime}\right) \beta_{1} \alpha_{2} \beta_{1}{ }^{*} \alpha_{2}{ }^{*} \\
& +P^{\beta \beta \beta \beta}\left(\rho_{1} \rho_{2} \mid \rho_{1}^{\prime} \rho_{2}^{\prime}\right) \beta_{1} \beta_{2} \beta_{1}, * \beta_{2}{ }^{*} \\
& +P^{\alpha \beta \beta \alpha}\left(\rho_{1} \rho_{2} \mid \rho_{1}^{\prime} \rho_{2}^{\prime}\right) \alpha_{1} \beta_{2} \beta_{1}{ }^{*} \alpha_{2}{ }^{*} \\
& +P^{\beta \alpha \alpha \beta}\left(\rho_{1} \rho_{2} \mid \rho_{1}^{\prime} \rho_{2}^{\prime}\right) \beta_{1} \alpha_{2} \alpha_{1}, * \beta_{2}, *
\end{aligned}
$$

when the wavefunctions are eigenfunctions of the $z$ component of the total spin operator. We need symmetric operators which will give us the various spatial components shown in Eq. (5). McWeeny and Mizuno have shown this may be accomplished with the use of an integral operator. We take an alternative approach and define a translation operator $T_{i}(\rho)$ with the properties

$$
T_{i}(\rho) u\left(r_{1}, r_{2}, \cdots, r_{n}\right)=U\left(r_{1}, \cdots, r_{i}+\rho, \cdots, r_{n}\right),
$$

where $U$ is any square integrable function of $n$ sets of spatial coordinates. Such an operator is unitary. Using $T_{i}(\rho)$ and the Dirac delta function we see that

$$
\begin{aligned}
P_{\mathrm{og}}^{\alpha \alpha} & =\sum_{i=1}^{n} \delta\left(r_{i}-\rho_{1}^{\prime}\right) T_{i}\left(\rho_{1}-\rho_{1}^{\prime}\right)\left(\frac{1}{2}+S_{z i}\right) \\
& =\sum_{i=1}^{n} Q_{i}\left(\frac{1}{2}+S_{z i}\right)
\end{aligned}
$$

gives

$$
\left\langle\Phi\left|P_{\mathrm{op}}^{\alpha \alpha}\right| \Phi\right\rangle /\langle\Phi \mid \Phi\rangle=P^{\alpha \alpha}\left(\rho_{1} \mid \rho_{1}{ }^{\prime}\right)
$$

\footnotetext{
${ }^{6}$ F. A. Matsen and A. A. Cantu, J. Phys. Chem. 72, 21 (1968).

7 R. McWeeny, Proc. Roy. Soc. (London) A253, 242 (1959)

${ }^{8} \mathrm{R}$. McWeeny and Y. Mizuno, Proc. Roy. Soc. (London) A254, 554 (1960).
}

The last factor in the terms on the right of Eq. (7) is in the form given by McWeeny and Mizuno. It is observed that the binominal coefficient in the definition Eq. (2) is produced by $P_{o p}{ }^{\alpha \alpha}$ since there are $\left(\begin{array}{l}n \\ 1\end{array}\right)$ terms in Eq. (7). The operator $P_{\mathrm{op}}^{\beta \beta}$ is given by

$$
P_{\mathrm{op}}^{\beta \beta}=\sum_{i=1}^{n} Q_{i}\left(\frac{1}{2}-S_{z i}\right) \text {. }
$$

For the second-order densities we have several forms to consider. Thus one obtains

$$
\begin{aligned}
& P_{\mathrm{op}}^{\alpha \alpha \alpha \alpha}=\frac{1}{2} \sum_{i<j} Q_{i j}+\left(\frac{1}{2}+S_{z i}\right)\left(\frac{1}{2}+S_{z j}\right), \\
& P_{\mathrm{op}}{ }^{\beta \beta \beta}=\frac{1}{2} \sum_{i<j} Q_{i j}+\left(\frac{1}{2}-S_{z i}\right)\left(\frac{1}{2}-S_{z j}\right), \\
& P_{\mathrm{op}}{ }^{++}=\frac{1}{2} \sum_{i<j} Q_{i j}+\left(\frac{1}{4}-S_{z i} S_{z j}\right), \\
& P_{\mathrm{op}}{ }^{++^{\prime}}=\frac{1}{4} \sum_{i<j} Q_{i j}{ }^{+}\left(S_{i}{ }^{+} S_{j}^{-}+S_{i}-S_{j}^{+}\right), \\
& P_{\mathrm{op}}{ }^{-}=\frac{1}{2} \sum_{i<j} Q_{i j}-\left(S_{z i}-S_{z j}\right), \\
& P_{\mathrm{op}}{ }^{--^{\prime}}=\frac{1}{4} \sum_{i<j} Q_{i j}^{-}\left(S_{i}^{+} S_{j}^{-}-S_{i}^{-} S_{j}^{+}\right),
\end{aligned}
$$

where

$$
\begin{aligned}
Q_{i j}^{ \pm}= & \delta\left(\boldsymbol{r}_{i}-\rho_{1}^{\prime}\right) \delta\left(\boldsymbol{r}_{j}-\rho_{2}^{\prime}\right) T_{i}\left(\rho_{1}-\rho_{1}^{\prime}\right) T_{j}\left(\rho_{2}-\rho_{2}{ }^{\prime}\right) \\
& \pm \delta\left(\boldsymbol{r}_{i}-\rho_{2}^{\prime}\right) \delta\left(\boldsymbol{r}_{j}-\rho_{1}^{\prime}\right) T_{i}\left(\rho_{2}-\rho_{2}{ }^{\prime}\right) T_{j}\left(\rho_{1}-\rho_{1}{ }^{\prime}\right)
\end{aligned}
$$

and

$$
\begin{aligned}
& P_{\mathrm{op}}^{\alpha \beta \alpha \beta}=P_{\mathrm{op}}{ }^{++}+P_{\mathrm{op}}-- \\
& P_{\mathrm{op}}{ }^{\beta \alpha \beta \alpha}=P_{\mathrm{op}}++-P_{\mathrm{op}}-{ }^{-}, \\
& P_{\mathrm{op}}{ }^{\alpha \beta \beta \alpha}=P_{\mathrm{op}}{ }^{+}+P_{\mathrm{op}}{ }^{-}, \\
& P_{\mathrm{op}}{ }^{\beta \alpha \alpha \beta}=P_{\mathrm{op}}{ }^{+\prime}-P_{\mathrm{op}}{ }^{-} .
\end{aligned}
$$

Each of the operators given by Eq. (10) is totally symmetric with respect to permutations of the subscripts.

It is seen that each of the first-order density operators is in the form $\sum_{i} A_{i} B_{i}$, and that each of the secondorder density operators is in the form $\sum_{i<j} A_{i j} B_{i j}$, where $A$ and $B$ refer to spatial and spin coordinates, respectively. In the latter case $A_{i j}= \pm A_{j i}$ and $B_{i j}= \pm B_{i i}$, where the upper or lower signs apply together. We must analyse these forms to determine some of their algebraic properties. We take up the first-order density first.

It is easily shown ${ }^{9}$ that a set of $n$ quantities $A$ $A_{1}, A_{2}, \cdots, A_{n}$ form a basis for a reducible representation of $S_{n}$ if the permutations operate on the subscripts. This representation is a direct sum of the irreducible representations $[n]$ and $[n-1,1]$. We may form linear combinations of the $A$ 's which belong to these repre-

\footnotetext{
M. Hamermesh, Group Theory (Addison-Wesley Publ. Co., Inc., Reading, Mass., 1962).
} 
sentations. Let us denote the totally symmetric function $A^{(0)}$ and the $n-1$ functions belonging to

$$
[n-1,1], A_{1}{ }^{(1)}, A_{2}{ }^{(1)}, \cdots, A_{n-1}{ }^{(1)} \text {. }
$$

The functions are considered to be real and algebraically. normalized, that is, the sum of the squares of the coefficients in each is equal to one. If we form a similar set from the operators $B_{i}$ it is seen that

$$
\sum_{i=1}^{n} A_{i} B_{i}=A^{(0)} B^{(0)}+\sum_{i=1}^{n-1} A_{i}{ }^{(1)} B_{i}^{(1)}
$$

The precise form of Eq. (13) is obtained only if $A_{i}{ }^{(1)}$ and $B_{i}{ }^{(1)}$ are arranged to give identical representations of $[n-1,1]$, and this is assumed. Explicite expressions are possible and one such is given by

$$
\begin{aligned}
& A^{(0)}=n^{-1 / 2} \sum_{i} A_{i} \\
& A_{i}^{(1)}=[i(i+1)]^{-1 / 2}\left(\sum_{j=1}^{i} A_{j}-i A_{i+1}\right), \quad i=1,2, \cdots, n-1,
\end{aligned}
$$

with the same expressions for the $B$ 's. The form of Eq. (13) is convenient for our purposes since each of the operators in the sum has a definite symmetry.

Similarly, a set of quantities $A_{i j}=A_{j i} ; i \neq j$ form the basis of a representation which in reduced form is $[n]+[n-1,1]+[n-2,2]$. The dimension of $[n-2,2]$ is $n(n-3) / 2$. If $A^{(0)}, A_{i}{ }^{(1)}$, and $A_{i}{ }^{(2)}$ are bases for these three irreducible representations, respectively, and $B^{(0)}, B_{\imath}{ }^{(1)}$, and $B_{i}{ }^{(2)}$ are a similar set from the $B^{\prime}$ s we have

$$
\sum_{i<j} A_{i j} B_{i j}=A^{(0)} B^{(0)}+\sum_{i} A_{i}{ }^{(1)} B_{i}{ }^{(1)}+\sum_{i} A_{i}{ }^{(2)} B_{i}{ }^{(2)} .
$$

When $A_{i j}=-A_{j i} ; i \neq j$, these quantities are a basis for a representation equivalent to $[n-1,1]+\left[n-2,1^{2}\right]$, and an expression similar to Eq. (15) may be written. The dimension of $\left[n-2,1^{2}\right]$ is $(n-1)(n-2) / 2$.

\section{DENSITY MATRICES}

We may consider each of the terms on the right of Eqs. (13) or (15) separately, and the portions belonging to $[n]$ are trivial since these operators satisfy all of the requirements that a spin-free operator does. Therefore, one obtains from Eq. (4)

$$
\begin{aligned}
& \frac{\left\langle\Phi\left|A^{(0)} B^{(0)}\right| \Phi\right\rangle}{\langle\Phi \mid \Phi\rangle} \\
& \quad=\frac{\left\langle\phi\left|A^{(0)} E^{(\mu)}(\gamma)\right| \phi\right\rangle\left\langle\omega\left|B^{(0)} E^{(\bar{\mu})}(\gamma)\right| \omega\right\rangle}{\left\langle\phi\left|E^{(\mu)}(\gamma)\right| \phi\right\rangle\left\langle\omega\left|E^{(\bar{\mu})}(\gamma)\right| \omega\right\rangle} .
\end{aligned}
$$

In this case $A^{(0)}$ is just $n^{-1 / 2}$ times the first-order spinless density operator or $[2 / n(n-1)]^{1 / 2}$ times the corre-
TABLE I. Symmetric spin operators.

\begin{tabular}{ll}
\hline & \multicolumn{1}{c}{$B^{(0)}$} \\
\hline$P^{\alpha \alpha}$ & $\frac{1}{2} n^{1 / 2}+n^{-1 / 2} S_{z}$ \\
$P^{\beta \beta}$ & $\frac{1}{2} n^{1 / 2}-n^{-1 / 2} S_{z}$ \\
$P^{\alpha \alpha \alpha \alpha}$ & {$\left[\frac{1}{4}[n(n-2)]+(n-1) S_{z}+S_{z}{ }^{2}\right] x$} \\
$P^{\beta \beta \beta \beta}$ & {$\left[\frac{1}{4}[n(n-2)]-(n-1) S_{z}+S_{z}{ }^{2}\right] x$} \\
$P^{++}$ & {$\left[\frac{1}{4} n^{2}-S_{z}^{2}\right] x$} \\
$P^{++1}$ & {$\left[2 S^{2}-2 S_{2}^{2}-n\right] x$} \\
& $x=[8 n(n-1)]^{-1 / 2}$ \\
\end{tabular}

sponding second-order operator. Table I gives the spin parts for each of the cases.

We now consider an operator term of the form $\sum_{i} A_{i}{ }^{(\alpha)} B_{i}{ }^{(\alpha)}$. It is shown in II that the $\gamma^{\prime}$ s in $E^{(\mu)}(\gamma)$ may be included in the spatial function $\phi$ as an alternative to their being placed with $E^{(\mu)}(\gamma)$. Thus $E^{(\mu)}(\gamma)$ is proportional to

$$
\sum_{i, j} \gamma_{i} \gamma_{j} \sigma_{i}^{-1} N P N \sigma_{j}
$$

where the $\sigma_{i}$ are permutations which transform the principal standard Young tableau into the others. Thus Eq. (4) could be written

$$
\begin{aligned}
\Phi & =C \sum_{i=1}^{f_{\mu}}\left[e_{i 1}^{(\mu)} \phi(\gamma)\right]\left[e_{i 1}^{(\bar{\mu})} \omega\right] \\
& =C \sum_{i} \phi_{i}{ }^{(\mu)} \omega_{i}{ }^{(\bar{\mu})},
\end{aligned}
$$

where $e_{11}^{(\bar{\mu})}$ now is proportional to $N P N$. The operator $P N P$ corresponding to $(\mu)$ could be chosen also for $e_{11}^{(\mu)}$. The constant $C$ in Eq. (17) is to be adjusted so that $\phi_{i}{ }^{(\mu)}$ and $\omega_{i}{ }^{(\bar{\mu})}$ are algebraically normalized. For the $\omega_{i}^{(\mu)}$ this is true normalization since these functions are written in an orthonormal basis. Using Eq. (17), (13), and (15) we obtain

$$
\begin{array}{r}
\left\langle\Phi\left|\sum_{i} A_{i}{ }^{(\alpha)} B_{i}{ }^{(\alpha)}\right| \Phi\right\rangle /\langle\Phi \mid \Phi\rangle=\left(f_{\mu}\left\langle\phi_{1}{ }^{(\mu)} \mid \phi_{1}{ }^{(\mu)}\right\rangle\right)^{-1} \\
\quad \times \sum_{i j / k}\left\langle\phi_{j}{ }^{(\mu)}\left|A_{i}{ }^{(\alpha)}\right| \phi_{k}{ }^{(\mu)}\right\rangle\left\langle\omega_{j}^{(\bar{\mu})}\left|B_{i}{ }^{(\alpha)}\right| \omega_{k}{ }^{(\bar{\mu})}\right\rangle .
\end{array}
$$

As is well known, linear combinations of quantities like $A_{i}{ }^{(\alpha)} \phi_{k}{ }^{(\alpha)}$ can be constructed which are bases for irreducible representations of $S_{n}$; thus

$$
\phi_{l}{ }^{(\gamma)^{\prime}}=\sum_{i k}\left(\alpha i_{\mu} k \mid \gamma l\right) A_{i}^{(\alpha)} \phi_{k}^{(\mu)},
$$

where the $\left(\alpha i_{\mu} k \mid \gamma l\right)$ are the Clebsch-Gordan coefficients. ${ }^{9}$ In general, one needs another index in these coefficients if any of the $(\gamma)$ appear more than once, but we will restrict the discussion to cases where Eq. (19) applies. The $(\alpha i \mu k \mid \gamma l)$ are elements of an orthogonal 
matrix; thus

$$
\sum_{i k}(\alpha i \mu k \mid \gamma l)\left(\alpha i \mu k \mid \gamma^{\prime} l^{\prime}\right)=\delta_{\gamma \gamma^{\prime}} \delta_{l l^{\prime}}
$$

and

$$
\sum_{\gamma l}(\alpha i \mu k \mid \gamma l)\left(\alpha i^{\prime} \mu k^{\prime} \mid \gamma l\right)=\delta_{i i^{\prime}} \delta_{k k^{\prime}} .
$$

Using Eqs. (19) and (21), one obtains

$$
A_{i}^{(\alpha)} \phi_{k}^{(\mu)}=\sum_{\gamma l}(\alpha i \mu k \mid \gamma l) \phi_{l}^{(\gamma) \prime}
$$

The case for $B_{i}{ }^{(\alpha)} \omega_{B}{ }^{(\bar{\mu})}$ may appear more complicated since these functions are not all linearly independent because $B_{i}{ }^{(\alpha)} \omega_{h}{ }^{(\bar{\mu})}$ is still a sum of simple products of spin functions. This means that some of the functions defined by Eq. (19) are identically zero, and these relations give the connections between the $B_{i}{ }^{(\alpha)} \omega_{k}(\bar{\mu})$. Nevertheless, Eq. (22) still applies-some of the terms in the sum are zero; thus

$$
B_{i}^{(\alpha)} \omega_{k}^{(\bar{\mu})}=\sum_{\gamma, l}(\alpha i \bar{\mu} k \mid \bar{\gamma} l) \omega_{l}^{(\bar{\mu}) \prime} .
$$

In Eq. (23) one of the symmetry relations of ClebschGordon coefficients has been used, viz.,

$$
(\alpha i \mu k \mid \gamma l)=(\alpha i \bar{\mu} k \mid \bar{\gamma} l),
$$

where the bar indicates the conjugate representation. Using Eqs. (22)-(24) we obtain

$$
\sum_{i k}\left(A_{i}^{(\alpha)} \phi_{k}^{(\mu)}\right)\left(B_{i}^{(\alpha)} \omega_{k k}^{(\bar{\mu})}\right)=\sum_{\gamma l} \phi_{l}^{(\mu)^{\prime}} \omega_{l}^{(\bar{\mu}) \prime},
$$

and

$$
\frac{\left\langle\Phi\left|\sum_{i} A_{i}^{(\alpha)} B_{i}{ }^{(\alpha)}\right| \Phi\right\rangle}{\langle\Phi \mid \Phi\rangle}=\frac{\left\langle\phi_{1}^{(\mu)} \mid \phi_{1}^{(\mu)^{\prime}}\right\rangle\left\langle\omega_{1}^{(\bar{\mu})} \mid \omega_{1}^{(\bar{\mu})^{\prime}}\right\rangle}{\left\langle\phi_{1}^{(\mu)} \mid \phi_{1}^{(\mu)}\right\rangle}
$$

If $(\alpha i \mu 1 \mid \mu 1)=(\alpha i \bar{\mu} 1 \mid \bar{\mu} 1) \neq 0$ we may solve Eq. (22) for $\phi_{1}{ }^{(\mu) \prime}$ and correspondingly $\omega_{1}^{(\bar{\mu}) \prime}$ and the right side of Eq. (26) becomes

$$
\frac{1}{\langle\alpha i \mu 1| \mu 1)^{2}} \frac{\left\langle\phi_{1}^{(\mu)}\left|A_{i}^{(\alpha)}\right| \phi_{1}^{(\mu)}\right\rangle\left\langle\omega_{1}^{(\bar{\mu})}\left|B_{i}^{(\alpha)}\right| \omega_{1}^{(\bar{\mu})}\right\rangle}{\left\langle\phi_{1}^{(\mu)} \mid \phi_{1}{ }^{(\mu)}\right\rangle},
$$

and we have the desired result, an expression involving only $e_{11}{ }^{(\mu)}$ (and $e_{11}{ }^{(\bar{\mu})}$ ). The crucial step in our derivation is the assumption that $(\alpha i \mu 1 \mid \mu 1) \neq 0$, of course, and as will be shown this fails for the case $\alpha=\left[n-2,1^{2}\right]$. Thus we cannot obtain the complete second-order density by this method. However, we can obtain the symmetric part, and this is sufficient for an operator with a symmetric spatial part.

Another noteworthy aspect of our result is that only one function each of the types $A_{i}{ }^{(\alpha)}$ and $B_{i}{ }^{(\alpha)}$ is needed, that is, we need only $e_{i i}{ }^{(\alpha)}$ and no other part of the algebra.

\section{CLEBSCH-GORDON COEFFICIENTS AND SPIN OPERATOR INTEGRALS}

As is well known, relations between $(\alpha i \beta j \mid \gamma k)$ and the irreducible representation matrix elements may be obtained. In our special case the square is obtained directly:

$$
(\alpha 1 \beta 1 \mid \beta 1)^{2}=\left(f_{\beta} / g\right) \sum_{\pi} D^{(\beta)}(\pi)_{11}^{2} D^{(\alpha)}(\pi)_{11} .
$$

It is shown in $I$ that the representations $\beta$ are of the type $\left[2^{(n / 2)-S^{\prime}}, 1^{2 S}\right]$ and $\bar{\beta}$ are $[(n / 2)+S,(n / 2)-S]$, where $n$ is the number of electrons and $S$ is the total spin quantum number. Because of the symmetry of Eq. (28) with respect to $\beta$ and $\bar{\beta}$ we may use either, and we arbitrarily use $\bar{\beta}$, writing $[n-k, k]$ where $k=\frac{1}{2} n-S$. Figure 1 gives the numbering that we use of the positions in the tableau $[n-k, k]$. Since the inclusion of the $\gamma$ 's (in $\phi$ ) in the treatment guarantees the generality of the results, any $e_{11}{ }^{(k)}$ may be used. As appears to be usually the case, $P N P$ (for spin functions!) is simpler, therefore we base most of our analysis on this form.

It is shown in II that

$$
D^{(\beta)}(\pi)_{11}=D^{(\beta)}(l)_{11} D^{(\beta)}\left(\pi_{B}\right)_{11},
$$

where $\pi_{B} \in P$ and $l$ is one of the left coset generators for the subgroup $P$. Hence, Eq. (28) will be most easily evaluated if both the representations $(\alpha)$ and $(\beta)$ have the same base subgroup $P$. Now $(\beta)$ is one of the representations $[n-k, k]$, whereas $(\alpha)$ is $[n-1,1]$, $[n-2,2]$, or $\left[n-2,1^{2}\right]$. Therefore, Littlewood's theorem (I) indicates this is possible for $[n-1,1]$, $k \geq 1$ and $[n-2,2], k \geq 2$. The representation $\left[n-2,1^{2}\right]$ must be treated differently. We take up the operators of the first-order density matrix first.

Using standard methods ${ }^{9}$ it is easily shown that

$$
\begin{array}{r}
{[n-1,1] \times[n-k, k]=[n-k-1, k+1]+[n-k, k]} \\
+[n-k+1, k-1]+[n-k, k-1,1] \\
+[n-k-1, k, 1], \quad n \neq 2 k \\
=[k+1, k-1]+[k, k-1,1], \\
n=2 k . \quad(30)
\end{array}
$$

The portions of Eq. (30) consisting of three-part partitions do not occur in the application since $B_{1}{ }^{(1)} \omega_{k}{ }^{(\beta)}$ is still a sum of product spin functions, and spin func-

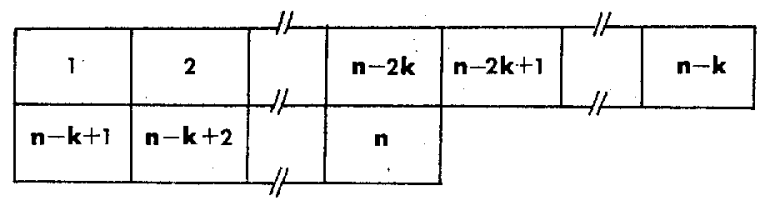

FIG. 1. The general two-row tableau for spin functions, $(\bar{\mu})$. 
tions corresponding to these representations do not exist. Equation (30) also gives the result obtained in a different way by McWeeny and Mizuno, that the first-order density operator applied to a spin eigenfunction of quantum number $S$ gives states of $S+1, S$, and $S-1$. In order to satisfy the requirement that the base subgroup for $(\alpha)$ and $(\beta)$ in Eq. (28) be the same, it is sufficient (and necessary) that $B_{1}{ }^{(1)}$ be an eigenfunction with eigenvalue +1 of every $\pi \in P$.

It is easily seen that

$$
B^{(1)}=B_{1}-B_{n-k+1}
$$

is a function belonging to $[n-1,1]$ and taking

$$
B_{1}^{(1)}=\text { const. } \times P\left(B_{1}-B_{n-k+1}\right)
$$

satisfies the above requirement. In order to make the notation more compact, we define $U$ as the set of integers in the first (upper) row of the tableau in Fig. 1 and $L$ as the integers in the second (lower) row and define

$$
\begin{aligned}
& B_{U}=\sum_{i \in U} B_{i}, \\
& B_{L}=\sum_{i \in L} B_{i} .
\end{aligned}
$$

Then one obtains

$$
B_{1}^{(1)}=\left[k B_{U}-(n-k) B_{L}\right] /[n k(n-k)]^{1 / 2}
$$

for the algebraically normalized operator. Equations (28) and (32) with the results of II yield

$$
([n-1,1] 1[n-k, k] 1 \mid[n-k, k] 1)^{2}=(n-k+1)(n-2 k) /(n-k)(n-2 k+2), \quad k \geq 1 .
$$

When we have quantities of the type $B_{i j}= \pm B_{j i}$, these too support the representation $[n-1,1]$. We define

$$
\begin{aligned}
& B_{U V}=\sum_{i<j \in U} B_{i j}, \\
& B_{U L}=\sum_{i \in U} \sum_{j \in L} B_{i j,} \\
& B_{L L}=\sum_{i<j \in L} B_{i j},
\end{aligned}
$$

and the $[n-1,1]$ function may be written in terms of these quantities. For the case $B_{i j}=B_{j i}$ we have

$$
B_{1}^{(1)}=\left[2 k B_{U U}-(n-2 k) B_{U L}-2(n-k) B_{L L}\right] /[n k(n-2)(n-k)]^{1 / 2},
$$

and for the $B_{i j}=-B_{j i}$, we have

The matrix element

$$
B_{1}^{(1)}=B_{U L} /[n(n-k)]^{1 / 2} \text {. }
$$

$$
\left\langle\omega_{1}^{(\bar{\mu})}\left|B_{1}^{(1)}\right| \omega_{1}^{(\bar{\mu})}\right\rangle
$$

may now be worked out. This is a straightforward but tedious calculation and the results are given in Table II.

The calculation for the $[n-2,2]$-type operators possesses a complication. It may be shown, in general, that

$$
\begin{array}{r}
{[n-2,2] \times[n-k, k]=[n-k+2, k-2]+[n-k+1, k-1]+2[n-k, k]+[n-k-1, k+1]+[n-k-2, k+2]} \\
+(\text { three- and four-part partitions), } \quad 2 \leq k \leq n-k-2, \quad
\end{array}
$$

and it is seen that $[n-k, k]$ appears twice in the reduced form. This introduces much complexity into our treatment for the density (but the transition densities do not have the difficulty) and this case shall not be discussed in general. Equation (34) does not apply to singlet or doublet states, and we see that

$[n-2,2] \times[n-k, k]=[k+3, k-2]+[k+2, k-1]+[k+1, k]+$ (three- and four-part partitions), $\quad n=2 k+1$

$$
=[k+2, k-2]+[k, k]+\text { (three- and four-part partitions), } \quad n=2 k .
$$

Therefore, we may apply our treatment to these states with" no change. We shall not discuss the higher multiplicity cases.

The operator corresponding to the $[n-2,2]$ representation constructed from doubly indexed quantities $B_{i j}=B_{j i}$ is easily seen to be

$$
B_{1}^{(2)}=\frac{\sqrt{2}\left[k(k-1) B_{U U}-(n-k-1)(k-1) B_{U L}+(n-k)(n-k-1) B_{L L}\right]}{[(n-1)(n-2) k(k-1)(n-k)(n-k-1)]^{1 / 2}} .
$$


With the results given in II and Eq. (37) we may calculate the appropriate Clebsch-Gordon coefficient, and one gets

$$
\begin{aligned}
& ([n-2,2] 1[n-k, k] 1 \mid[n-k, k] 1)^{2} \\
& \quad=\frac{k(k-1)(n-2 k+2)(n-2 k+3)-2(n-1)(k-1)(n-2 k+1)(n-2 k+3)+(n-1)(n-2)(n-2 k+1)(n-2 k+2)}{(n-k-1)(n-k)(n-2 k+2)(n-2 k+3)} .
\end{aligned}
$$

Of course, Eq. (38) gives the sum of the squares of two Clebsch-Gordon coefficients for multiplicities greater than doublet. Table III gives the values of the integral

$$
\left\langle\omega_{1}^{(\bar{\mu})}\left|B_{1}^{(2)}\right| \omega_{1}^{(\bar{\mu})}\right\rangle
$$

for the various $B_{i j \text {. }}$

The operator $P^{-{ }^{-}}$contains spin operators of the property $B_{i j}=-B_{j i}$ which leads to the representation $[n-1,1]+\left[n-2,1^{2}\right]$, as we have seen. The results for $[n-1,1]$ have already been given, but the representation $\left[n-2,1^{2}\right]$ requires a new technique since, as we have seen, we cannot construct an $e\left[n-2,1^{2}\right]_{11}$ with the same base subgroup as those giving the spin and space functions.

First, we see that

$$
\begin{aligned}
& {\left[n-2,1^{2}\right] \times[n-k, k]} \\
& \quad=[n-k+1, k-1]+[n-k, k]+[n-k-1, k+1] \\
& \quad+(3-\text { and 4-part partions), } \cdot k \leq n-k-2, \quad
\end{aligned}
$$

in general, and the special cases of doublet and singlet states give

$$
\begin{aligned}
{\left[n-2,1^{2}\right] \times[n-k, k] } & \\
& =[k+1, k]+[k+2, k-1], \quad n=2 k+1 \\
& =[k+1, k-1], \quad n=2 k .
\end{aligned}
$$

TABLE II. Integrals for $[n-1,1]$, singly and doubly indexed spin operators.

\begin{tabular}{ll}
\hline \multicolumn{1}{c}{$B_{i}$ or $B_{i j}$} & \multicolumn{1}{c}{$\left\langle\omega_{1}^{\left.(\bar{\mu})\left|B_{1}{ }^{(1)}\right| \omega_{1}(\bar{\mu})\right\rangle}\right.$} \\
\hline$\frac{1}{2} \pm S_{z i}$ & $\pm x$ \\
$\left(\frac{1}{2}+S_{z i}\right)\left(\frac{1}{2}+S_{z j}\right)$ & $(n-k-1)(n-k+1) y$ \\
$\left(\frac{1}{2}-S_{z i}\right)\left(\frac{1}{2}-S_{z j}\right)$ & $-(k-1)(n-k+1) y$ \\
$\frac{1}{4}-S_{z i} S_{z j}$ & $\frac{1}{2}(n-k+1)(n-2 k) y$ \\
$S_{i}+S_{j}-+S_{i}-S_{j}^{+}$ & $2(n-k) y$ \\
$S_{i}{ }^{+} S_{j}^{-}-S_{i}-S_{j}^{+}$ & 0 \\
$S_{z i}-S_{z j}$ & $x$ \\
& $x=\frac{(n-k+1)(n-2 k)}{(n-2 k+2)}\left[\frac{k}{n(n-k)}\right]^{1 / 2}$ \\
& $y=\frac{(n-2 k)}{(n-2 k+2)\left[\frac{k}{n(n-2)(n-k)}\right]^{1 / 2}}$ \\
\hline \hline
\end{tabular}

Equations (40) and (41) have three- and four-part partitions omitted. The difficulties attendent to the $[n-2,2]$ representation do not arise here, and we see, also, that Eq. (41) implies the density component $P^{-{ }^{-}}$is zero for singlet states.

Let us chose any three of the numbers $1,2, \cdots, n$ : $i, j$, and $k$. The operator

$$
B_{i j k}{ }^{(3)}=B_{i j}+B_{j k}+B_{k i}
$$

belongs to the $\left[n-2,1^{2}\right]$ representation, and an orthonormal set of representation functions may be written as linear combinations of the $B_{i j k}^{(3)}$. It is easy to show that

$$
\left\langle\omega_{1}^{(\bar{\mu})}\left|B_{i j k}^{(3)}\right| \omega_{1}^{(\bar{\mu})}\right\rangle=0
$$

for all choices of $i, j, k$, thus this type of integral is zero for all $\left\langle\omega_{1}^{(\bar{\mu})}\left|B_{1}{ }^{(3)}\right| \omega_{1}{ }^{(\bar{\mu})}\right\rangle$, and nonzero integrals must be of the type $\left\langle\omega_{j}^{(\bar{\mu})}\left|B_{i}^{\left({ }^{(3)}\right.}\right| \omega_{1}(\bar{\mu})\right\rangle, j \neq 1$. If we let $a=$ $n-k+1$ the Appendix shows how we may obtain an operator $e_{21}^{(\bar{\mu})}$ from $e_{11}^{(\bar{\mu})}$. We get

$e_{21}^{(\bar{\mu})}=[(n-k+1)(n-k-1)]^{-1 / 2}[I+(n-k)(1 a)] e_{11}{ }^{(\mu)}$, and if we take $B_{1}{ }^{(3)}=B_{12 a}{ }^{(3)}$,

$$
\begin{aligned}
\left(\left[n-2,1^{2}\right] 1\right. & {[n-k, k] 1 \mid[n-k, k] 2)^{2} } \\
& =(f / g) \sum_{\pi} D^{(3)}(\pi)_{11} D^{(\bar{\mu})}(\pi)_{22} D^{(\bar{\mu})}(\pi)_{11}
\end{aligned}
$$

is zero only for the singlet case. Equation (43) may be evaluated as the others, and we find

$$
(n-2 k)(n-k+1)^{2} / 3 k(n-2 k+2)(n-k-1)^{3},
$$

and for the spin integral we get

$$
\begin{aligned}
\left\langle\omega_{2}{ }^{(\bar{\mu})}\right. & \left.\left|B_{1}{ }^{(3)}\right| \omega_{1}{ }^{(\bar{\mu})}\right\rangle \\
= & (n-2 k)(n-k+1)^{3 / 2} / \sqrt{3}(n-2 k+2)(n-k-1)^{3 / 2} .
\end{aligned}
$$

\section{THE REDUCED OPERATORS FOR DENSITY- MATRIX COMPONENTS}

We may now determine the components of the density matrices with the results of the previous sections. Using $D$ to represent any of the density operators from 
TABLE III. Integrals for $[n-2,2]$, doubly indexed spin operators.

\begin{tabular}{|c|c|}
\hline$B_{i j}$ & $\left\langle\omega_{1}^{(\mu)}\left|B_{1}^{(2)}\right| \omega_{1}^{(\mu)}\right\rangle$ \\
\hline$\left(\frac{1}{2} \pm S_{z i}\right)\left(\frac{1}{2} \pm S_{z j}\right)$ & $\begin{array}{l}{[k(k-1)(n-2 k+2)(n-2 k+3)-2(n-1)(k-1)(n-2 k+1)(n-2 k+3)} \\
\quad+(n-1)(n-2)(n-2 k+1)(n-2 k+2)] x\end{array}$ \\
\hline$S_{i}{ }^{+} S_{j}^{-}+S_{i}-S_{j}{ }^{+}$ & $\begin{array}{l}2[(3 n-2 k+3)(n-k+1)(n-2 k+1)-2 k] x \\
x=[(n-2 k+2)(n-2 k+3)]^{-1}[k(k-1) / 2(n-1)(n-2)(n-k)(n-k-1)]^{1 / 2}\end{array}$ \\
\hline
\end{tabular}

Eqs. (13) or (15), (19), and (27), we get

$\frac{\langle\Phi|D| \Phi\rangle}{\langle\Phi \mid \Phi\rangle}=\sum_{(\alpha)} \frac{\left\langle\phi_{1}^{(\mu)}\left|A_{1}^{(\alpha)}\right| \phi_{1}{ }^{(\mu)}\right\rangle\left\langle\omega_{1}^{(\bar{\mu})}\left|B_{1}^{(\alpha)}\right| \omega_{1}^{(\bar{\mu})}\right\rangle}{(\alpha 1 \mu 1 \mid \mu 1)^{2}\left\langle\phi_{1}^{(\mu)} \mid \phi_{1}^{(\mu)}\right\rangle}$,

except for the $\left[n-2,1^{2}\right]$ representation from $Q_{i j}^{-}$, which case is taken up later. Equation (46) may be used to define a reduced operator

$$
R=\sum_{\alpha}\left[\left\langle\omega_{j}^{(\bar{\mu})}\left|B_{1}^{(\alpha)}\right| \omega_{1}^{(\bar{\mu})}\right\rangle /(\alpha 1 \mu 1 \mid \mu 1)^{2}\right] A_{1}^{(\alpha)},
$$

and it is seen that

$$
\langle\Phi|D| \Phi\rangle /\langle\Phi \mid \Phi\rangle=\left\langle\phi_{1}^{(\mu)}|R| \phi_{1}^{(\mu)}\right\rangle /\left\langle\phi_{1}^{(\mu)} \mid \phi_{1}^{(\mu)}\right\rangle .
$$

We list in Table IV the reduced operators for the components of the first-order density matrix, and in Table $V$ the expressions for singlet and doublet states components for the second-order density matrix. In these tables the same convention is used concerning the $U$ and $L$ subscripts as is used in Eqs. (31b) and (34a).

It can be seen immediately from Table $V$ that the linear relation

$$
P^{\alpha \alpha \alpha \alpha}+P^{\beta \beta \beta \beta}=2 P^{++}+P^{++}
$$

holds. This has been observed for singlet states by Kutzelnig. ${ }^{10}$ It is seen that the same result holds for doublet states.

The $P_{\mathrm{op}}{ }^{-{ }^{\prime}}$ is somewhat different as was pointed out above. The $[n-1,1]$ part is zero as can be seen from Table II. The $\left[n-2,1^{2}\right]$ portion gives as a reduced operator

$$
\begin{array}{r}
R=-[k(n-k)(n-k-1) /(n-k+1)](1 a) \\
\times\left(Q_{12}-+Q_{2 a}-+Q_{a 1}-\right),
\end{array}
$$

TABLE IV. Reduced operators for $\gamma\left(x_{1} x_{1}{ }^{\prime}\right), M_{8}=S$.

\begin{tabular}{cc}
\hline \hline$D$ & $R$ \\
\hline$P_{\mathrm{op}}^{\alpha \alpha}$ & $Q_{U}$ \\
$P_{\mathrm{op}}{ }^{\beta \beta}$ & $Q_{L}$ \\
\hline
\end{tabular}

${ }^{10}$ W. Kutzelnig, Z. Naturforsch. 18a, 1058 (1963). where we have included the effect of the shifting operator $e_{21}{ }^{(\mu)}$ in $R$. This operator yields

$P^{-\prime}\left(\rho_{1} \rho_{2} \mid \rho_{1}^{\prime} \rho_{2}^{\prime}\right)=\left\langle\phi_{1}^{(\mu)}|R| \phi_{1}{ }^{(\mu)}\right\rangle /\left\langle\phi_{1}^{(\mu)} \mid \phi_{1}{ }^{(\mu)}\right\rangle$,

but we may reduce the numerator of Eq. (51) to some extent. The $(\mu)$ in Eq. (51) corresponds to the tableau conjugate to that shown in Fig. 1, and since

we have

$$
\phi_{1}^{(\mu)}=e_{11}{ }^{(\mu)} \phi
$$

$$
\left\langle\phi_{1}{ }^{(\mu)}|R| \phi_{1}{ }^{(\mu)}\right\rangle=\left\langle\phi_{1}{ }^{(\mu)}\left|\nu^{-1} R \nu\right| \phi_{1}{ }^{(\mu)}\right\rangle,
$$

where $\nu$ is any permutation of $N$ for $(\mu)$ [or any permutation of $P$ for $(\bar{\mu})]$. It should be noted that $(1 a)$ is not one of these permutations. We average Eq. (53) over this subgroup of $S_{n}$ and obtain

$$
\begin{aligned}
&\left\langle\phi_{1}{ }^{(\mu)}|R| \phi_{1}{ }^{(\mu)}\right\rangle=[k !(n-k) !]^{-1}\left\langle\phi_{1}(\mu)\left|\sum_{\nu} \nu^{-1} R \nu\right| \phi_{1}{ }^{(\mu)}\right\rangle \\
& \sum_{\nu} \nu^{-1} R \nu=(k-1) !(n-k-2) ! \\
& \times \sum_{i<j \in U} \sum_{k \in L}[(i k)-(j k)]\left(Q_{i j}-+Q_{j k}-+Q_{k i}-\right) \\
&=(k-1) !(n-k-2) ! \bar{R}
\end{aligned}
$$

and therefore,

$\left\langle\phi_{1}{ }^{(\mu)}|D| \phi_{1}{ }^{(\mu)}\right\rangle=-(n-k+1)^{-1}\left\langle\phi_{1}{ }^{(\mu)}|\bar{R}| \phi_{1}^{(\mu)}\right\rangle$.

That Eq. (55) represents a simplification is seen since $N$ for $(\mu)$ is a factor of $e_{11}{ }^{(\mu)}$ and this factor commutes with $\bar{R}$, and hence may be removed from the expression.

TABLE V. Reduced operators for $\Gamma\left(x_{1} x_{2} \mid x_{1}{ }^{\prime} x_{2}{ }^{\prime}\right)$; $M_{\mathrm{s}}=S, S=0, \frac{1}{2}$.

\begin{tabular}{ll}
\hline \multicolumn{1}{c}{$D$} & \multicolumn{1}{c}{$R$} \\
\hline$P_{\mathrm{op}}{ }^{\alpha \alpha \alpha \alpha}$ & $(1 / 2) Q_{U V^{+}}$ \\
$P_{\mathrm{op}}{ }^{\beta \beta \beta \beta}$ & $(1 / 2) Q_{L L}{ }^{+}$ \\
$P_{\mathrm{oD}}{ }^{++}$ & $(1 / 2) Q_{U L^{+}}$ \\
$P_{\mathrm{oD}}{ }^{++}$ & $\frac{1}{4}\left[Q_{U V^{+}}-Q_{U L^{+}}+Q_{L L}{ }^{+}\right]$ \\
$P_{\mathrm{oD}^{-}}{ }^{--}$ & $(1 / 2) Q_{U L}{ }^{-}$ \\
$P_{\mathrm{oD}}{ }^{-1}$ & Discussed in text \\
\hline
\end{tabular}


TABLE VI. Reduced operators for components of $\gamma\left(x_{1} \mid x_{1}^{\prime}\right)$ direct and transitions.

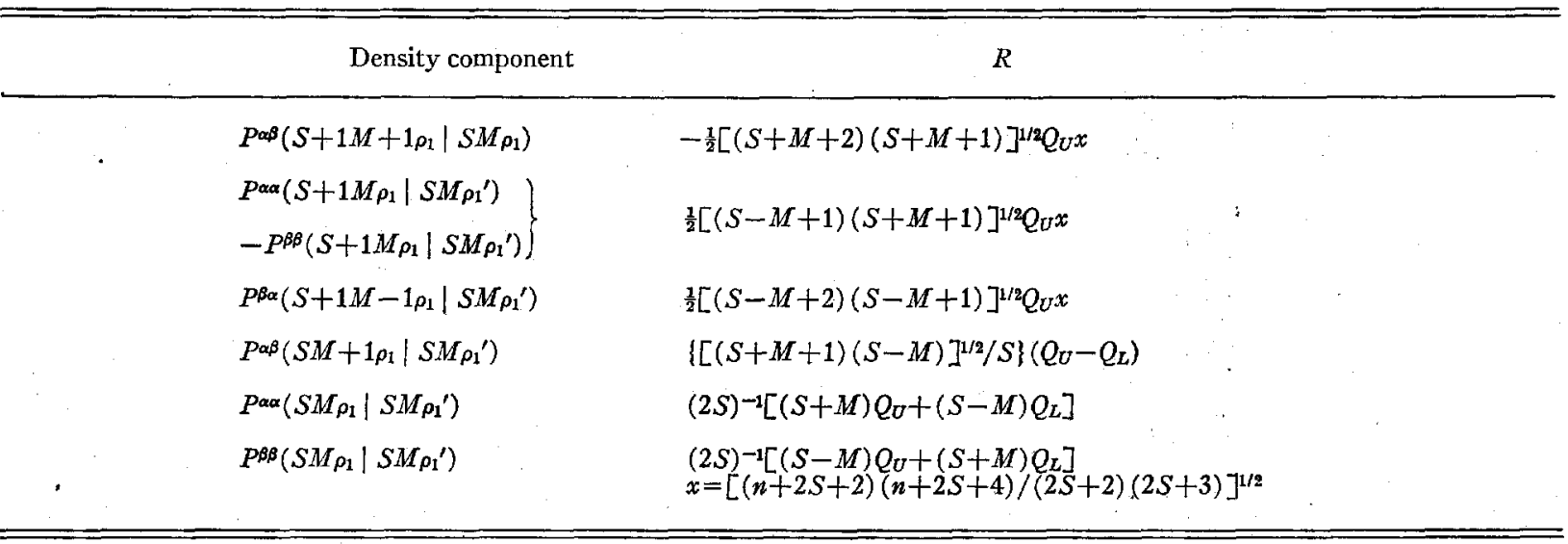

This places the evaluation of $P_{\mathrm{op}}{ }^{-1}$ on the same basis as the other operators we have obtained.

The reduced operators for the first-order density matrix given in Table IV apply only to the principal case $M_{*}=S$. We may calculate the other cases and also the transition densities following the above procedure, and with the use of the raising and lowering operators for $M_{s}$. Table VI gives the complete set of reduced operators for the first-order density operators. The cases not listed are obtainable from those given. Care needs to be used since these formulas are appropriate for $Q_{U}$ and $Q_{L}$ based on the tableau shape of $S$ and not upon that for $S+1$. In addition, another ClebschGordon coefficient is needed for those components involving $S$ and $S+1$. Since $S$ is associated with $[n-k, k]$ and $S+1$ is associated with $[n-k+1, k-1]$, we obtain

$$
\begin{aligned}
([n-1,1] 1[n-k, k] 1 \mid & {[n-k+1, k-1] 1)^{2} } \\
= & n / k(n-k+1)(n-k) .
\end{aligned}
$$

It is easily seen from Table VI that we obtain the same result for the spin density as that given in Ref. 6.

\section{APPENDIX: AN OPERATOR $e_{21}^{(\mu)}$}

From the general properties of group algebras it is known that

$$
\begin{aligned}
e_{21}{ }^{(\mu)} e_{11}{ }^{(\mu)} & =e_{21}{ }^{(\mu)}, \\
e_{11}{ }^{(\mu)} e_{21}{ }^{(\mu)} & =0, \\
e_{21}{ }^{(\mu) \dagger} e_{21}{ }^{(\mu)} & =e_{12}{ }^{(\mu)} e_{21}{ }^{(\mu)}=e_{11}{ }^{(1 \mu)} .
\end{aligned}
$$

If $\pi$ is any permutation with the property

$$
e_{11}^{(\mu)} \pi e_{11}^{(\mu)}=D^{(\mu)}(\pi)_{11} e_{11}^{(\mu)} \neq \pm e_{11}^{(\mu)} \text {, or } 0
$$

it is seen that

$$
y \equiv\left[D^{(\mu)}(\pi)_{11} I-\pi\right] e_{11}^{(\mu)}
$$

satisfied Eqs. (A1) and (A2). Equation (A3) may be used to normalize $y$; thus

$$
\begin{aligned}
y^{\dagger} y & =e_{11}{ }^{(\mu)}\left[D^{(\mu)}(\pi)_{11} I-\pi^{-1}\right]\left[D^{(\mu)}(\pi)_{11} I-\pi\right] e_{11}{ }^{(\mu)} \\
& =\left[1-D^{(\mu)}(\pi)_{11^{2}}{ }^{2} e_{11}^{(\mu)},\right.
\end{aligned}
$$

and we have used the fact that $D^{(\mu)}(\pi)_{11}=D^{(\mu)}\left(\pi^{-1}\right)_{11}$ for real representations. Therefore, $e_{21}^{(\mu)}$ may be taken as

$$
y /\left[1-D^{(\mu)}(\pi)_{11^{2}}\right]^{1 / 2} .
$$

If $\pi$ is a simple binary interchange $(i, j)$ which connects the two rows of the tableau in Fig. $1(i \in U, j \in L)$, then

and

$$
D^{(\bar{\mu})}[(i, j)]_{11}=-(n-k)^{-1},
$$

$e_{21}^{(\bar{\mu})}=[I+(n-k)(i, j)] e_{11}^{(\bar{\mu})} /[(n-k+1)(n-k-1)]^{1 / 2}$, and

$$
e_{21}^{(\mu)}=[I-(n-k)(i, j)] e_{11}^{(\mu)} /[(n-k+1)(n-k-1)]^{1 / 2}
$$

for the tableau conjugate to that of Fig. 1. 